\title{
Photonic Integrated Microwave Phase Shifter up to the mm-Wave Band With Fast Response Time in Silicon-on-Insulator Technology
}

\author{
Claudio Porzi ${ }^{\circledR}$, Giovanni Serafino, Marc Sans, Fabio Falconi ${ }^{\circledR}$, Vito Sorianello, Sergio Pinna ${ }^{\circledR}$, John E. Mitchell, \\ Marco Romagnoli, Antonella Bogoni, and Paolo Ghelfi (1)
}

\begin{abstract}
An integrated silicon-on-insulator microwave photonic phase shifter is demonstrated, based on an optical deinterleaver providing box-like transfer function and a reverse-biased pn-junction waveguide optical phase shifter. The photonic integrated circuit is proved to precisely control the phase of microwave signals in a range of more than $400^{\circ}$ with a fast reconfiguration time of $1 \mathrm{~ns}$, with a broad bandwidth of more than $6 \mathrm{GHz}$ around an RF carrier flexibly selectable between 10 and $16 \mathrm{GHz}$, and limited in-band RF power variations. Moreover, thanks to the periodic behavior of the deinterleaver, the device is demonstrated to correctly control the phase of signals in the mm-wave band $(>30$ GHz) as well. The performance guaranteed by the proposed microwave phase shifter suggests its use in demanding applications as rapidly reconfiguring phased array antennas in future wireless communications systems.
\end{abstract}

Index Terms-Microwave photonics, phase shifters, photonic integrated circuits, silicon photonics.

\section{INTRODUCTION}

I N MANY microwave and RF systems, phase shifters (PSs) are important components; For instance, they are required for controlling the relative phase between the elements of a phased array antenna in radars or in steerable communications links, or

Manuscript received February 2, 2018; revised April 28, 2018; accepted May 21, 2018. Date of publication June 11, 2018; date of current version August 30, 2018. This work was supported by the Initial Training Network, FiWiN5G, funded by the H2020 Marie Curie Actions of the European Commission (H2020MSCA-ITN-2014 N642355). (Corresponding author: Claudio Porzi.)

C. Porzi and G. Serafino are with the TeCIP Institute, Scuola Superiore Sant'Anna, Pisa 56124, Italy (e-mail: c.porzi@sssup.it; g.serafino@sssup.it).

M. Sans and J. E. Mitchell are with the Department of Electronic and Electrical Engineering, University College London, London WC1E 6BT, U.K. (e-mail: m.sans@ucl.ac.uk; j.mitchell@ucl.ac.uk).

F. Falconi, V. Sorianello, M. Romagnoli, and P. Ghelfi are with the Photonic Networks and Technologies National Laboratory, Consorzio Nazionale Interuniversitario per le Telecomunicazioin, Pisa 56124, Italy (e-mail: fabio.falconi@cnit.it; vito.soranello@cnit.it; marco.romagnoli@cnit.it; paolo. ghelfi@cnit.it).

S. Pinna is with the TeCIP Institute Scuola Superiore Sant'Anna, Pisa 56124, Italy, and also with the Electrical and Computer Engineering Department, University of California, Santa Barbara, CA 93106 USA (e-mail: pinna@ece.ucsb.edu).

A. Bogoni is with the TeCIP Institute, Scuola Superiore Sant'Anna, Pisa 56124, Italy, and also with the Photonic Networks and Technologies National Laboratory, Consorzio Nazionale Interuniversitario per le Telecomunicazioin, Pisa 56124, Italy (e-mail: a.bogoni@sssup.it).

Color versions of one or more of the figures in this paper are available online at http://ieeexplore.ieee.org.

Digital Object Identifier 10.1109/JLT.2018.2846288 for managing the signal phase in electronic warfare systems, in microwave instrumentation, and in measurement systems.

With the ability of naturally mapping optical phase shifts into RF phase shifts, microwave photonics (MWP) has been proposed since several years as a possible mean to overcome the limitations of electronic circuits for PS implementation [1]-[16]. In particular, besides being immune to electromagnetic interferences, photonics potentially offers the additional advantages of broadband operation at large RF carrier values, high phase accuracy with small amplitude variations, and fast response time, which are all key features for several emerging applications, including those in forthcoming $5 \mathrm{G}$ systems. Among the different proposed techniques, solutions based on photonic integrated circuits (PICs) distinguished as the most promising approach for practical MWP-based PS implementation, due to increased stability, drastically minimized size and weight, and possibility of low operating power. By resorting to different technological platforms, several PICs have been realized in the last years to implement microwave PS operation. The first proposed solutions exploited Mach-Zehnder (MZ) modulators to implement either incoherent I-Q vector modulation technique [2] or coherent phase signal processing [1], [3]. Improved variations of the coherent approach have been more recently reported in [4] and [9]. All these implementations were realized in either $\mathrm{LiNbO}_{3}$ or polymer waveguides, and relied on the integration of several electro-optic modulators. Furthermore, such platforms do not support the fabrication of active elements such as photodiodes for fully-functional PICs implementation and, in case of $\mathrm{LiNbO}_{3}$, fabricated devices exhibit a relatively large footprint of few $\mathrm{cm}^{2}$. Another approach was proposed based on ultrafast stimulated Brillouin scattering in nonlinear chalcogenide waveguides [10]. Large optical power levels are in this case required to trigger the nonlinearity. Furthermore, the maximum RF operating frequency is limited to twice the Brillouin frequency shift of the medium, which is $\sim 7.6 \mathrm{GHz}$ in chalcogenide glasses [10]. Applying thermo-optic effect in silica [5], silicon [6], [7], and $\mathrm{Si}_{3} \mathrm{~N}_{4}$ [8] microring resonators (MRRs), is another largely exploited technique in order to control the phase of a microwave signal in the optical domain. In [13], a PIC realized in siliconon-insulator (SOI) technology that employs a deinterleaver filter based on a MZ interferometer, and a thermally tunable optical PS in a silicon waveguide has also been discussed. Thermal effects are however slow, and typically hinder operations above 


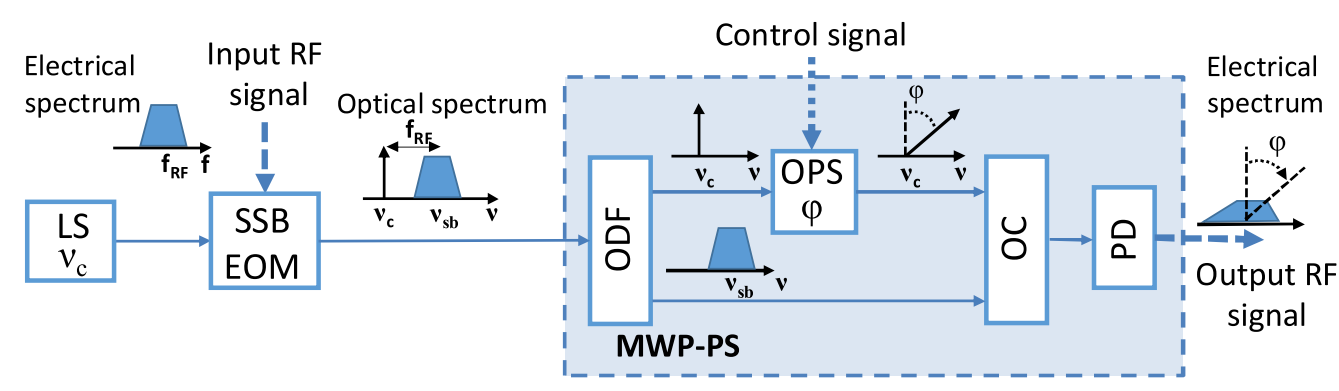

(a)

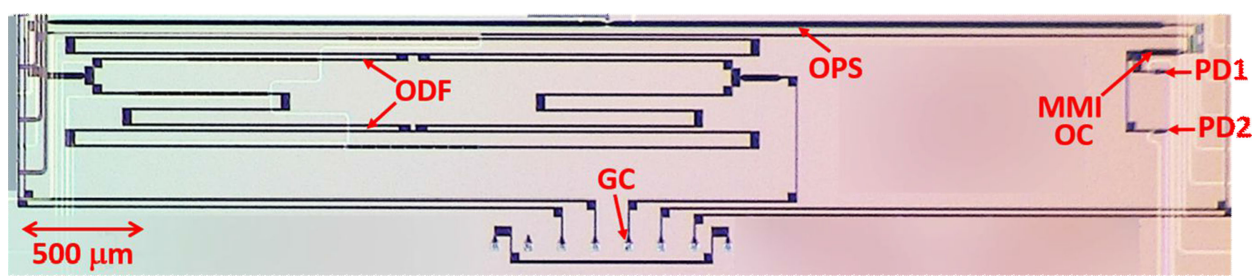

(b)

Fig. 1. (a) Schematic and operation principle of the proposed photonic integrated phase shifter. LS: laser source; SSB EOM: single-sideband electro-optical modulator; ODF: optical deinterleaver filter; OPS: optical phase shifter; OC: optical coupler; PD: photodiode. In the scheme, $f_{R F}$ represents the RF signal carrier frequency, $\nu_{c}$ the optical carrier frequency, $\nu_{s b}$ is the central optical frequency of the optical SSB modulated signal, $\varphi$ is the variable optical/RF phase shift. (b) Picture of the fabricated PIC implementing the MWP-PS; GC: grating coupler; MMI-OC: multi-mode interference optical coupler. Two PDs are connected to the two MMI outputs. As shown by the scale indication, the PIC occupies an area of $\sim 5 \times 2.4 \mathrm{~mm}^{2}$.

$\sim 100 \mathrm{kHz}$. Another reported approach relying on SOI technology, is based on a dual phase-shifted Bragg grating realized in a silicon waveguide [10], where the tuning of a laser wavelength is proposed for modifying the phase of a microwave signal. The maximum achievable phase shift is however below $360^{\circ}$, and the resonant nature of the phase response limits the flexibility on the choice of the RF carrier.

The SOI technological platform, which notably permits to realize PICs with a CMOS-compatible process, also allows to exploit the free-carrier modulation effect [14], as a much faster mean (albeit weaker and with higher loss) than thermo-optic effect for controlling the phase of an optical signal. This has been exploited in [12], where a MRR formed by a p-i-n junction waveguide for carrier density modulation has been used to realize PS operation. However, a full $360^{\circ}$ phase shift requires to cascade multiple MRRs, and the intrinsic loss of the free carrier-modulation effect is exacerbated by the resonant configuration, producing large power variations around the operating wavelength translating into RF-dependent loss. Other recently proposed ultrafast approaches exploits complementary phased shifted spectra [15], and nonlinear phase amplification [16] techniques.

In this work, we present a photonic-integrated microwave PS realized in SOI technology which simultaneously matches the demand for wide phase-shift range, broad bandwidth, low in-band power oscillations, fast (i.e., sub- $\mu \mathrm{s}$ ) reconfiguration speed, and compatibility with CMOS integrated circuits fabrication technology. The PIC is able to perform stable phase shifts well in excess of $360^{\circ}$ over a bandwidth of $6 \mathrm{GHz}$ for RF carriers spanning in the $\mathrm{X}, \mathrm{Ku}$, an $\mathrm{mm}$-wave bands, with limited in-band power variations of $\sim 1 \mathrm{~dB}$, and small response time below $1 \mathrm{~ns}$.

\section{Photonic Integrated Circuit Operation}

The schematic structure and the operation principle of the proposed MWP-PS are illustrated in Fig. 1(a). The core element of the architecture, highlighted in the dashed box of the figure, comprises an optical deinterleaver filter (ODF), an optical phase shifter (OPS), an optical coupler (OC), and a photodiode (PD). At the input of the circuit, the microwave signal to be phaseshifted, which is considered to show a given power spectral density around the carrier frequency $f_{\mathrm{RF}}$, drives a single-sideband electro-optic modulator (SSB-EOM) to generate a sideband centered at the optical frequency $\nu_{s}$, spaced by $f_{R F}$ from the optical carrier at frequency $\nu_{c}$ provided by a laser source (LS). The generated full-carrier SSB modulated optical signal is then fed into the MWP-PS.

The operation of the scheme relies on optical carrier-sideband separation performed by the ODF and subsequent phase shift of the isolated carrier in the OPS stage, before the two components are recombined in the OC and sent to the PD to generate the phase-shifted microwave signal [13]. The phase of the down-converted signal at the PD output generated by the beating between the carrier and the sideband components is indeed given by the phase difference of the two beating signals. Assuming a perfect carrier-sideband isolation at the ODF outputs, the amount of optical phase shift $\varphi$ experienced by the optical carrier in the OPS translates into an equal phase shift $\varphi$ of the microwave signal at $f_{R F}$ without any amplitude modulation, as depicted in Fig. 1(a).

A PIC implementing the scheme within the dashed box of Fig. 1(a), has been designed and fabricated in SOI technology through a multi-project wafer run [17]. A microscope picture of the fabricated SOI PIC is shown in Fig. 1(b). To inject the optical signals into the photonic circuit, a grating coupler (GC) 


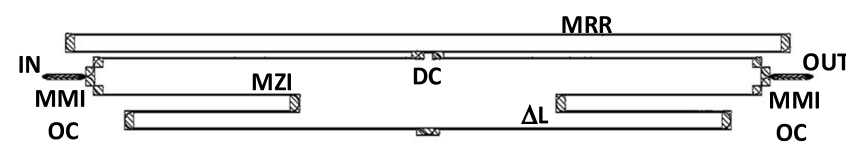

(a)

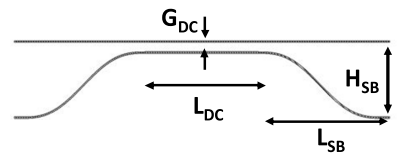

(b)

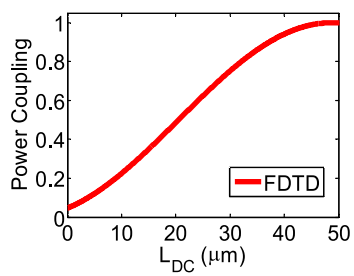

(c)
Fig. 2. (a) Mask layout of the fabricated optical deinterleaver filter (ODF); MZI: Mach-Zehnder interferometer; MRR: microring resonator; MMI OC: multimode interference optical coupler; DC: directional coupler. (b) Details of DC mask layout; Design parameters: DC gap, $\mathrm{G}_{\mathrm{DC}}$, DC length $\mathrm{L}_{\mathrm{DC}}$, sine bend (SB) length, $\mathrm{L}_{\mathrm{SB}}$, and height, $\mathrm{H}_{\mathrm{SB}}$; (c) Finite-difference time domain (FDTD) simulations of the DC power coupling coefficient vs. $\mathrm{L}_{\mathrm{DC}}$.

is used for vertical coupling with the accessing optical fiber. The ODF is implemented by a MRR-loaded MZ interferometer [18]. The OPS element is realized in a $4.25 \mathrm{~mm}$-long interdigitated $p-n$ junction, embedded in a silicon rib waveguide. The desired phase shift is achieved by applying the proper reverse voltage to the junction, which causes a variation in the extension of the depletion region. The corresponding change of the waveguide effective index allows for controlling the phase of the optical signal at the output of the OPS. A $2 \times 2$ multi-mode interference (MMI) OC recombines the phase-shifted optical carrier and the modulated sideband. By exploiting the two available phase-offset replica of the microwave signal, two $p-i-n$ germanium PDs, with a $-3 \mathrm{~dB}$ bandwidth of $\sim 20 \mathrm{GHz}$, have been connected to each output waveguide the $2 \times 2$ MMI. This has been done with the aim of realizing a subsequent off-chip balanced configuration for the PDs on a printed circuit board, by properly connecting the ground and signal contacts of the PD pairs, which has the twofold advantage of a $6 \mathrm{~dB}$ improvement in the output RF power and common mode noise cancellation.

The ODF has been designed to implement a 3rd-order halfband elliptic filter with a target minimum stopband attenuation of $30 \mathrm{~dB}$ [19]. The mask layout of the fabricated ODF is shown in Figure 2(a). Besides the $2 \times 2$ MMI OCs at the MZI input/output ports, all other structures are implemented with 450 nm-wide silicon strip waveguides. A directional coupler (DC) with sine bends $(\mathrm{SB})$ is used to partially couple the light in the shorter branch of the MZI with a MRR in racetrack configuration (the radius of the bent section is $30 \mu \mathrm{m}$ ). Details of the fabricated DC mask layout are also shown in Fig. 2(b), along with corresponding finite-difference time domain simulation of power coupling coefficient versus DC length in Fig. 2(c). All the relevant design parameters for the ODF are listed in Table I. Resistive heaters (not shown in the ODF mask layout), implemented with p-doped waveguides, have been placed in proximity of both MRR and MZI structures to realize a $\pi$ phase difference between the corresponding resonances for proper filter operation.
TABLE I

ODF DESIGN SUMMARY

\begin{tabular}{|c|c|c|c|c|c|}
\hline $\begin{array}{c}\text { MZI } \\
\text { delay } \\
\Delta \mathrm{L}\end{array}$ & $\begin{array}{c}\text { MRR } \\
\text { length }\end{array}$ & $\begin{array}{c}\text { DC } \\
\text { length } \\
\text { L }_{\mathrm{DC}}\end{array}$ & $\begin{array}{c}\text { DC gap } \\
\text { GDC }\end{array}$ & $\begin{array}{c}\text { SB } \\
\text { Length } \\
\text { LSB }_{\mathrm{SB}}\end{array}$ & $\begin{array}{c}\text { SB } \\
\text { Height } \\
\mathrm{H}_{\mathrm{SB}}\end{array}$ \\
\hline $2.7 \mathrm{~mm}$ & $2 \Delta \mathrm{L}$ & $33 \mu \mathrm{m}$ & $0.3 \mu \mathrm{m}$ & $20 \mu \mathrm{m}$ & $40 \mu \mathrm{m}$ \\
\hline
\end{tabular}

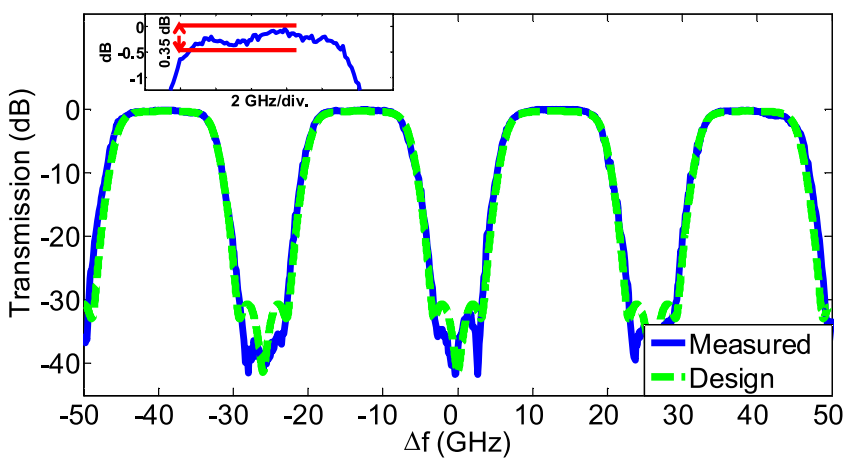

Fig. 3. Measured (solid blue line) transmission spectrum of the ODF, and corresponding design simulation (dashed green line) curves. The filter' free spectral range is $\sim 26 \mathrm{GHz}$; the $-1 \mathrm{~dB}$ bandwidth is $\sim 10 \mathrm{GHz}$. Inset: details of $\mathrm{a}-1 \mathrm{~dB}$ passband, showing typical amplitude ripple of less than $0.4 \mathrm{~dB}$.

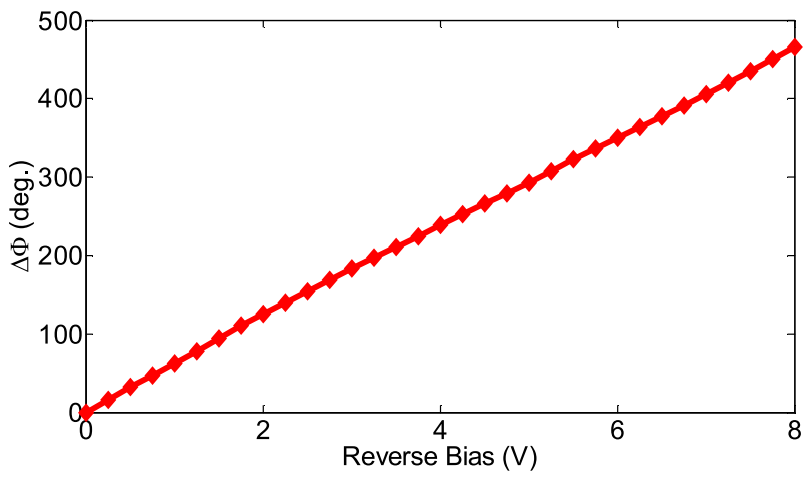

Fig. 4. Measured relative phase shift $\Delta \phi$ vs. applied reverse bias to the p-n junction waveguide OPS.

The optical transmission spectrum of the ODF at one output port, measured on a test structure, is depicted in Fig. 3. A periodic box-like transmission is observed, exhibiting a flat passband with a $-1 \mathrm{~dB}$ bandwidth of $\sim 10 \mathrm{GHz}$ and steep roll-off. The free-spectral-range (FSR) is $\sim 26 \mathrm{GHz}$, whereas the $-30 \mathrm{~dB}$ stop-band width is around $6.5 \mathrm{GHz}$. The fabricated ODF transmission output shows a very good agreement with the simulated target design, where a value of $\sim 1.7 \mathrm{~dB} / \mathrm{cm}$ has been used for the propagation loss of the strip waveguides, as available from the photonic design kit of the technological process. At the other ODF output, a complementary spectrum is retrieved. The OPS has also been characterized on a text structure, by embedding it within a MZ interferometer and observing the power variations of the output optical signal from the interferometer as a function of the applied reverse bias to the $p-n$ junction. The measured characteristic of the OPS is plotted in Fig. 4, where a linear $475^{\circ}$ phase shift over a bias range of $8 \mathrm{~V}$ for the $p$ - $n$ junction reverse bias is reported, giving the considerable precision of $0.06^{\circ} / \mathrm{mV}$. 


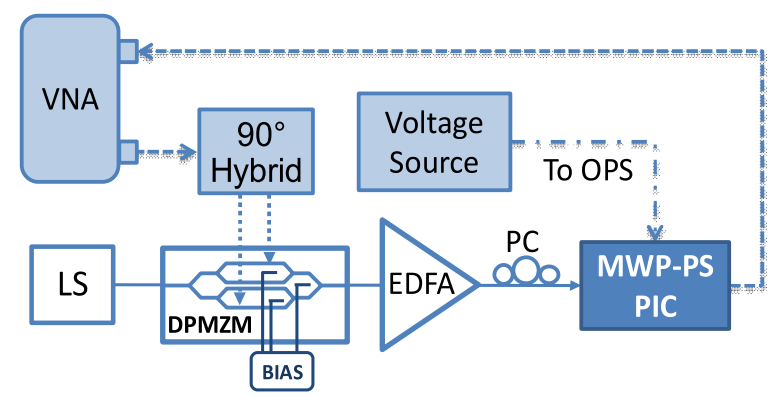

Fig. 5. Microwave photonic phase-shifter (MWP-PS) characterization experimental setup. LS: Laser Source; VNA: Vector Network Analyzer; DPMZM: dual parallel Mach-Zehnder modulator; EDFA: Erbium-Doped Fiber Amplifier; PC: Polarization Controller; PIC: Photonic Integrated Circuit; OPS: Optical Phase Shifter.

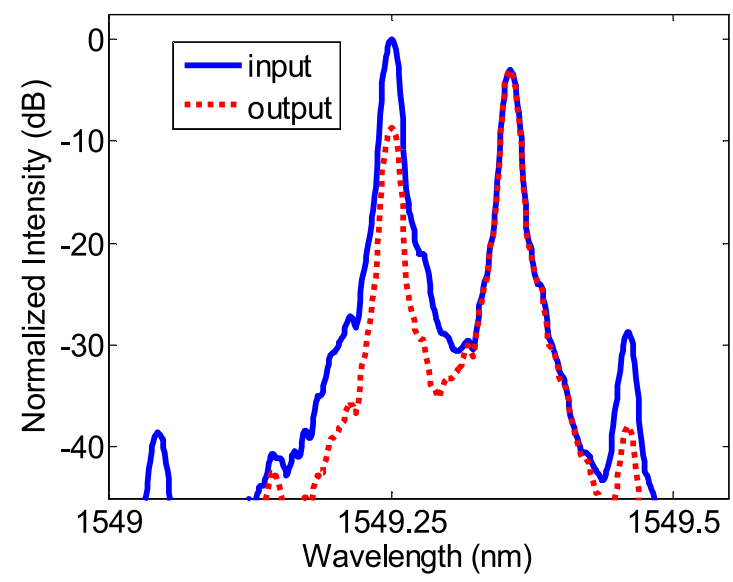

Fig. 6. Optical spectra of the SSB-modulated signal at PIC input/output for a $13 \mathrm{GHz}$ microwave frequency driving the modulator. Input spectrum (blue, solid line) is normalized to carrier power; output spectrum (red dotted line), taken at one output of the MMI $3 \mathrm{~dB}$ optical coupler on a sample structure without integrated PDs, is normalized to input upper sideband power.

\section{EXPERIMENTAL RESULTS}

The integrated MWP-PS has been characterized using the set-up of Fig. 5. A nested dual MZ modulator (NDMZM) has been used to generate a full-carrier SSB modulated optical signal from a LS. To this scope, a quadrature replica of the output frequency from a vector network analyzer (VNA) is generated through a $90^{\circ}$ hybrid coupler. The two signals are then fed to the two RF ports of the NDMZM, which is biased such to produce $90^{\circ}$ phase difference between the optical paths in the main MZ structure [20]. A typical spectrum of the signal at the NDMZM output is shown in Fig. 6 (blue solid line). After NDMZM, the generated SSB-modulated signal power is boosted by an erbium-doped fiber amplifier (EDFA) up to $\sim 10 \mathrm{dBm}$ and then injected into the MWP-PS PIC. Before entering the PIC, the optical signal passes through a polarization controller (PC) for maximizing the coupling efficiency of injected light with the fundamental TE mode of the silicon waveguides in the PIC through the GC. The optical carrier generated by the LS is tuned in the center of the ODF passband window by which it is routed towards the OPS branch of the MWP-PS (see Fig. 1(a)). Inside the PIC, the depletion region of $p-n$ junction embedded

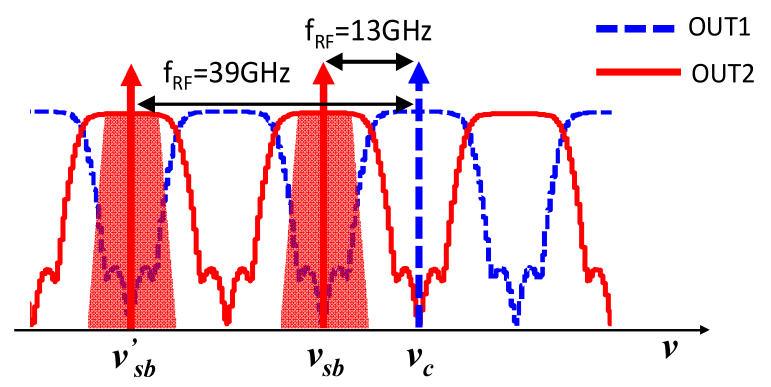

Fig. 7. Schematic representation of the position of the LS optical carrier $\nu_{\mathrm{c}}$ and sideband optical frequency $\nu_{\mathrm{sb}}$ with respect to ODF transfer functions OUT1 and OUT2 at its output ports for two exemplary values of the RF carrier $f_{R F}$.

in the OPS waveguide is controlled by a DC voltage source, operating the junction in reverse bias condition. To test the RF bandwidth of the proposed MWP-PS, the VNA has been operated in the sweep mode over a proper frequency range, so that the sideband optical frequency spanned across the ODF passband at the output port connected to the pass-through path of Fig. 1(a). The corresponding signal spectrum after the MMI coupler comprising the phase-shifted carrier and sideband, observed at one output of the $2 \times 2 \mathrm{MMI}$ on a test structure with GCs replacing the PD pairs, is also shown in Fig. 6 (red dotted line). From the figure, an attenuation of $\sim 12 \mathrm{~dB}$ for the carrier due to free-carrier absorption during propagation in the 4.25 $\mathrm{mm}$-long doped OPS waveguide can be observed. This value is in agreement with the value of $30 \mathrm{~dB} / \mathrm{cm}$ provided by the documentation of the technological process. The broadband strong carrier-to-sideband isolation of more than $30 \mathrm{~dB}$ provided by the ODF guarantees that the phase-shifted carrier entering the OC is about $20 \mathrm{~dB}$ higher than the residual un-suppressed carrier at the other input of the $\mathrm{OC}$, making interference effects at the coupler output negligible over a band in excess of $6 \mathrm{GHz}$. For the PIC used in the set-up of Fig. 5, the photodetected microwave signal produced by carrier-sideband beating in the PD is collected through an electrical probe and then sent back to the VNA for MWP-PS performance evaluation through magnitude and phase measurement of the $S_{21}$ parameter.

In a first set of measurements, the output continuous-wave frequency from the VNA is swept over a bandwidth of $6 \mathrm{GHz}$ around the central value of $f_{R F}=13 \mathrm{GHz}$. Therefore, the sideband of the full-carrier SSB modulated signal is swept over the transmission passband of the lower port of the ODF towards of Fig. 1(a). A sketch of the experiment description in the frequency domain is also shown in Fig. 7, where the position of the carrier and sideband optical frequencies relative to the ODF passband windows at its complimentary outputs for two relevant values of $f_{R F}$ are schematically illustrated. The corresponding measurements on $S_{21}$ parameters are reported in Fig. 8, where both the argument and phase of the $S_{21}$ parameters over the considered frequency span are reported for different values of the bias voltage applied to the OPS. By delivering the NDMZM output directly to a test PD in the PIC, a reference signal for both amplitude and phase response has been retrieved to balance out the frequency response of the NDMZM, $90^{\circ}$ hybrid, and PD. After reference calibration, the curves of the phase re- 


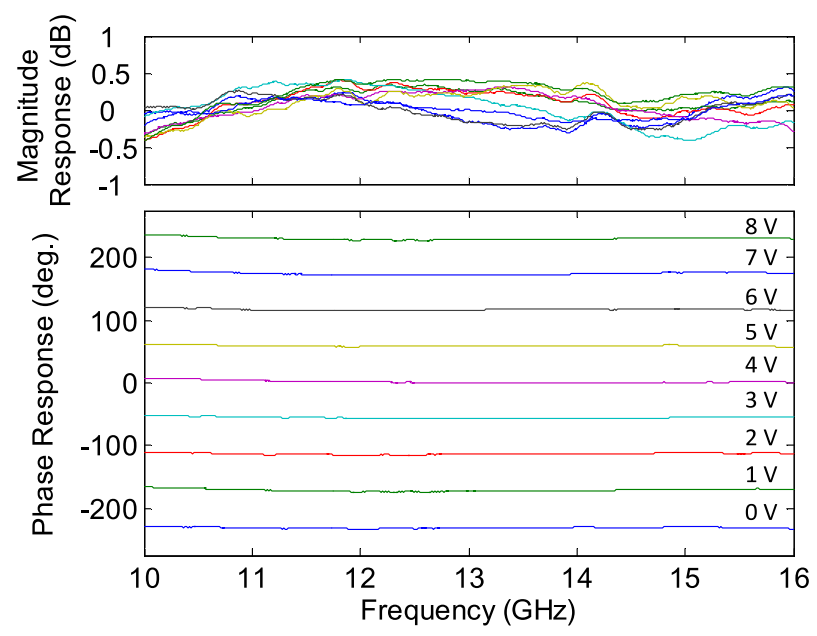

Fig. 8. Magnitude (top) and phase (bottom) response of the MWP-PS, as a function of the VNA output RF frequency, over a 10-to-16 GHz frequency span. The magnitude response traces are normalized to the average value. The phase response traces correspond to an applied bias voltages ranging (from top to bottom) between 0 and $-8 \mathrm{~V}$ with $-1 \mathrm{~V}$ steps. The voltage excursion ensuring a phase shift of $360^{\circ}$ corresponds to $\sim 6 \mathrm{~V}$.

sponse illustrate an almost flat phase shifts in the whole $6 \mathrm{GHz}$ band, spanning over more than $450^{\circ}$, as the OPS control signal ranges between 0 and $-8 \mathrm{~V}$. To compensate for additional delay introduced by the external circuitry and cables the VNA delay compensation function has been used, where the same value of the compensating delay has been used for all the traces. The standard deviation of the measured phase response in the full bandwidth range stays below $2^{\circ}$ over a $360^{\circ}$ phase shift. Due to the box-like ODF transmission, the power oscillations in the $6 \mathrm{GHz}$ span for all the considered voltage values are limited and well confined within $0.8 \mathrm{~dB}$, as illustrated in the normalized magnitude response curves. The average power variation of the $\mathrm{RF}$ signal for a $360^{\circ}$ phase shift, corresponding to $6 \mathrm{~V}$ control signal excursion, primarily due to the slightly voltage dependent loss characteristic of the pn-doped waveguide in the OPS, is $\sim 2.5 \mathrm{~dB}$. The amplitude variations of the RF signal for different phase-shift values can be eliminated using an additional variable attenuator stage in the PIC [13]. Similar characteristics have been observed by tuning the LS carrier frequency at the short- and long-frequency edge of the ODF passband, and performing a $6 \mathrm{GHz}$ sweep around $f_{R F}=10 \mathrm{GHz}$ and $f_{R F}=16 \mathrm{GHz}$, respectively, illustrating the potentials of the scheme for flexible operation with different RF carrier values. The corresponding fractional bandwidth of the photonic integrated microwave phase-shifter for RF carriers in the $\mathrm{X}$ to $\mathrm{Ku}$ bands is thus between $46 \%$ and $50 \%$, illustrating the broadband capabilities of the proposed approach.

As schematically illustrated in Fig. 7, the maximum and minimum frequencies at which the scheme operation is guaranteed, depend on the sharpness of the carrier-sideband separation filter. Thus, by designing a higher order ODF, the operation bandwidth could be improved. In the reported implementation, a single ring-loaded MZI, realizing 3rd-order elliptic half-band filter with minimum stopband attenuation of $30 \mathrm{~dB}$, has been

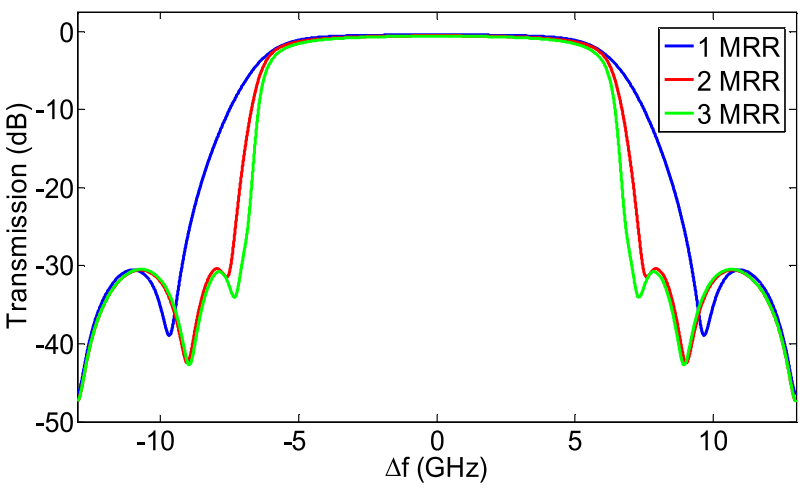

Fig. 9. Simulated design responses of the ODF implemented with single, double, and triple MRR-loaded MZI.

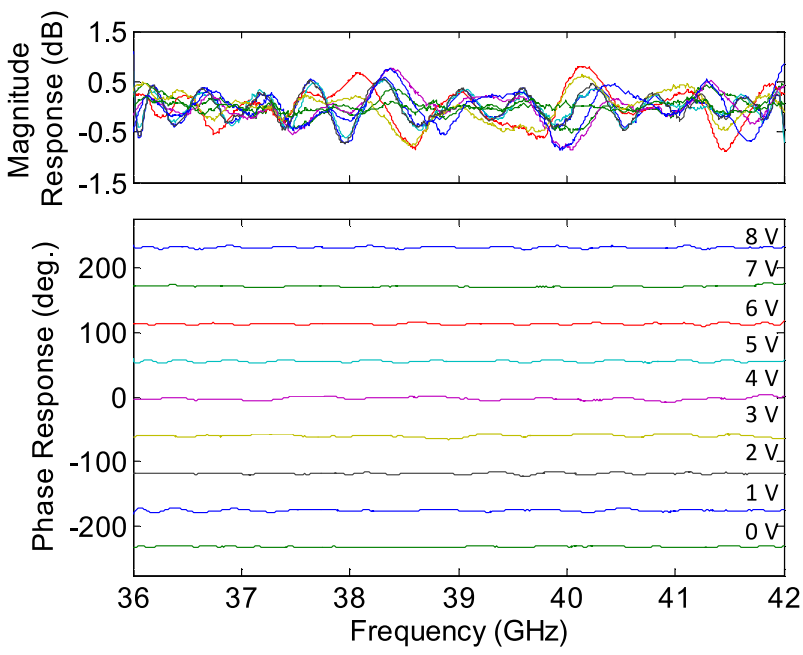

Fig. 10. Magnitude (top) and phase (bottom) response of the MWP-PS, as a function of the VNA output RF frequency, over a 36-to-42 GHz frequency span. The magnitude responses traces are normalized to the average value. The phase response traces correspond to an applied bias voltages ranging (from top to bottom) between 0 and $-8 \mathrm{~V}$ with $-1 \mathrm{~V}$ steps.

employed. The simulated transmission of the filter is compared in Fig. 9 with those of a two and a three rings-loaded MZI, corresponding to 5th and 7th order elliptic designs [19], respectively, with the same $30 \mathrm{~dB}$ minimum stopband attenuation. A propagation loss value of $1.7 \mathrm{~dB} / \mathrm{cm}$ has been used for all the simulations. As shown in the figure, the $-30 \mathrm{~dB}$ stopband is increased from about $8 \mathrm{GHz}$ in the single-ring case to around $11 \mathrm{~dB}$ in the double-ring MZI. A marginal improvement of less than $1 \mathrm{GHz}$ is obtained with the triple-ring architecture, which might not justify the increased complexity in the filter design and control systems.

The periodic response of the ODF reported in Fig. 3, where the FSR of $26 \mathrm{GHz}$ denotes the frequency spacing between consecutive passband windows, indicates that strong-rejection carrier-sideband isolation can be obtained around any RF carrier value that is an odd multiple of half the FSR. This property thus enables to suitably extend the described MWP-PS operation up to the mm-wave band, which is particularly appealing for several emerging applications, including high-speed wireless communications in 5G systems [21]. In order to asses mm-wave band 


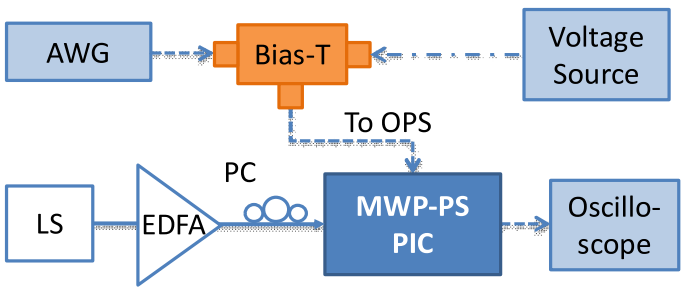

(a)

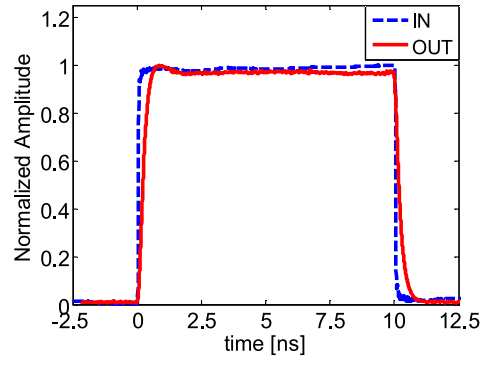

(b)

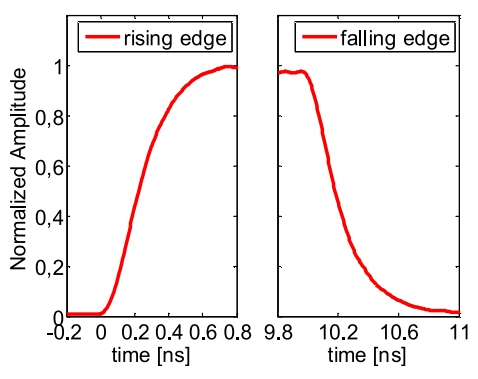

(c)

Fig. 11. (a) Experimental set-up for integrated MWP-PS step-response measurement. AWG: arbitrary waveform generator; LS: laser source; EDFA: erbiumdoped fibre amplifier; PC: polarization controller; PIC: photonic integrated circuit; OPS: optical phase shifter; MWP-PS: microwave-photonics phase shifter. (b) Oscilloscope traces of the amplitude-converted signal at PIC output (red, solid line) and square-wave signal applied to OPS (blues, dashed line). (c) Details of the rising (left) and falling (right) edge of the step-response reported in (b).

operation, the central value of the VNA frequency sweep has thus been set to $f_{R F}=39 \mathrm{GHz}$, as schematically illustrated in Fig. 7. Since the integrated germanium PD in the PIC provided a very limited response at such frequencies, a test structure with a GC for optical output access after the MMI coupler has been used in conjunction with an external PD with $-3 \mathrm{~dB}$ bandwidth of $35 \mathrm{GHz}$. The results of the measurements, which have been performed in a similar manner as in the case of Fig. 8, are reported in Fig. 10, illustrating the potentials of the proposed scheme for PS operation in the extremely high frequency (EHF) region. The measured RF power variations are within $\sim 1.5 \mathrm{~dB}$ over the considered frequency span in the whole $8 \mathrm{~V}$ voltage excursion, whereas the maximum standard deviation of the phase is around $3.5^{\circ}$. These fluctuations are partially ascribed also to the increased sensitivity of both the reference and sample traces to noise oscillations due to the limited bandwidth of the available electrical equipment in the set-up. For the case of $f_{R F}=39 \mathrm{GHz}$, the fractional bandwidth of the photonicintegrated microwave phase-shifter is reduced to $\sim 15 \%$, which is the trade-off for flexible selection of the operating RF band offered by the device. By specifically designing the ODF in order to provide a FSR of about $80 \mathrm{GHz}$ (which could be simply accomplished by changing the delay paths in the MZI and MRR comprising the ODF), a fractional bandwidth of about $50 \%$ around a target RF carrier of $40 \mathrm{GHz}$ would be possible. Generally speaking, in order to further increase the broadband operation of the circuit, an add/drop filter with bandwidth-toFSR ratio lower than $50 \%$, rather than the half-band ODF used in this implementation, could be adopted. Clearly, the sharpness and high contrast of the carrier/sideband separator filter should be preserved. To this scope, either high order serially coupled MRRs [22], MRR-loaded MZI structures [23], [24], or integrated high-order phase-shifted Bragg gratings [25] could be used.

\section{OPS RESPONSE TIME MEASUREMENT}

The interdigitated pn-junction implementing the OPS has been dimensioned to provide a phase shift in excess of $2 \pi$ with a reverse voltage below the junction breakdown voltage of $\sim 9 \mathrm{~V}$. A moderate doping level has been chosen to keep the propagation losses to within an acceptable level. These de- sign constrains resulted in a relatively long longitudinal junction waveguide of $4.5 \mathrm{~mm}$. In order to fully exploit the intrinsically large carrier-density modulation bandwidth (which is typically several tens of $\mathrm{GHz}$ in interdigitated or lateral $p$ - $n$ junction) in such long device, a travelling-wave electrode design would be required. However, in order to simplify circuit design, and reduce the overall layout footprint, a lumped-electrode approach has been chosen for the control signal responsible for depletionregion modulation, which resulted in a relatively large $\mathrm{RC}$ time constant. Nevertheless, this choice is justified by the fact that for practical PS application envisioned in $5 \mathrm{G}$ systems, a sub- $\mu \mathrm{s}$ response time can be accepted. In order to assess the response time of the fabricated OPS, the set-up reported in Fig. 11(a) has been employed. For this measurement, the output of a CW LS is directly amplified by an EDFA and launched into the MWP-PS PIC, after polarization adjustment through a PC. In particular the LS wavelength has been tuned in correspondence of the ODF transition bandwidth region, in order to split the incident light on both the ODF output ports such that the two signal replica recombines in the MMI-OC with similar amplitude. In such configuration, any change in the optical phase produced by the OPS translates into an intensity- modulated signal at the PD output. A large-signal analysis of the OPS modulation bandwidth has thus been performed by driving the $p-n$ junction waveguide with a step-function signal generated by an arbitrary waveform generator (AWG). A bias-tee has been used to couple the signal with the dc reverse voltage in order to prevent forward biasing of the junction. A square-wave signal with a peak amplitude of $2 \mathrm{~V}$, corresponding to a phase shift as large as $\sim 0.7 \pi$ has been used. The corresponding step-response of the OPS has been evaluated by monitoring the PD output signal on a sampling oscilloscope, as illustrated in Fig. 11(b) (red solid line), where the AWG output signal (blue dashed line) is also shown for comparison. The details of the rising and falling edges of the signal at the PIC output are also reported in Fig. 11(c), showing a fast settling time below $1 \mathrm{~ns}$.

\section{CONCLUSION}

A photonic integrated microwave phase-shifter has been realized in CMOS-compatible SOI technology. The device operation is based on carrier-sideband isolation from a full-carrier 
single-sideband modulated signal in an optical deinterleaver filter and on the processing of the optical carrier phase alone in an optical phase shifter. The circuit characterization confirmed wide phase shifts in excess of $360^{\circ}$ for a $6 \mathrm{~V}$ variation in the signal driving the optical phase shifter, with almost linear characteristic, corresponding to a precision below $0.1 \% \mathrm{mV}$. Broadband operation is ensured by the box-shaped optical deinterleaver feature, which enables high-rejection carrier-sideband isolation over a typical bandwidth of $6 \mathrm{GHz}$ and limits the inband RF power variations to within $1 \mathrm{~dB}$, besides improving the scheme stability against thermal and environmental fluctuations. The flat-top characteristic of the periodic deinterleaver passband window also permits flexible choice of the RF carrier over the X, $\mathrm{Ku}$, and $\mathrm{Q}$ bands, which illustrates the potentials of the scheme for photonic processing of signals for mm-wave communication systems. By designing the optical deinterleaver filter with proper FSR other microwave spectral bands can be covered as well. Additionally, by implementing the optical phase shifter in a p-n junction silicon rib waveguide exploiting fast carrierdepletion modulation effect, a small reconfiguration time of about $1 \mathrm{~ns}$ is achieved, which outperforms previous realizations in SOI technology based on thermo-optic effect. The relatively large insertion loss of the optical phase-shifter due to free-carrier absorption in doped waveguides could be eliminated using recently proposed approach for optical phase modulators based on ultra-fast DC Kerr effect in silicon [26]. The integration of germanium photodiodes provides improved chip functionality, by enabling the access of the phase-shifted microwave signal through electrical contacts, which ease the possible packaging of the device for practical system applications. The overall circuit footprint is $\sim 10 \mathrm{~mm}^{2}$. Further increased functionality could be achieved by on-chip integration of the single-sideband modulator as well. The scheme is therefore a suitable candidate for practical system applications such as rapidly-reconfiguring beam-steering in phased array antennas for future 5G systems.

\section{ACKNOWLEDGMENT}

The authors wish to thank Antonio D'Errico and Marzio Puleri from Ericsson Telecomunicazioni SpA, Italy, for fruitful discussions and support.

\section{REFERENCES}

[1] K. Matsumoto, M. Izutsu, and T. Sueta, "Microwave phase shifter using optical waveguide structure," J. Lightw. Technol., vol. 9, no. 11, pp. 15231527, Nov. 1991.

[2] J. F. Coward, T. K. Yee, C. H. Chalfant, and P. H. Chang, "A photonic integrated-optic RF phase shifter for phased array antenna beamforming applications," J. Lightw. Technol., vol. 11, no. 12, pp. 2201-2205, Dec. 1993.

[3] S.-S. Lee et al., "Demonstration of a photonically controlled RF phase shifter," IEEE Microw. Guided Wave Lett., vol. 9, no. 9, pp. 357-359, Sep. 1999.
[4] J. Han, B.-J. Seo, S. K. Kim, H. Zhang, and H. R. Fetterman, "Singlechip integrated electro-optic polymer photonic RF phase shifter array," $J$. Lightw. Technol., vol. 21, no. 12, pp. 3257-3261, Dec. 2003.

[5] D. B. Adams and C. K. Madsen, "A novel broadband photonic RF phase shifter," J. Lightw. Technol., vol. 26, no. 15, pp. 2712-2717, Aug. 2008.

[6] M. Pu et al., "Widely tuneable microwave phase shifter based on siliconon-insulator dual microring resonator," Opt. Express, vol. 18, no. 6, pp. 6172-6182, Mar. 2010

[7] G. Serafino et al., "Design and characterization of a photonic integrated circuit for beam forming in 5G wireless networks," in Proc. Int. Top. Meeting Microw. Photon., Beijing, China, 2017, pp. 1-4.

[8] M. Burla et al., "On-chip CMOS compatible reconfigurable optical delay line with separate carrier detuning for microwave photonic signal processing," Opt. Express, vol. 19, no. 22, pp. 21475-21484, Oct. 2011.

[9] E. H. W. Chan, W. W. Zhang, and R. A. Minasian, "Photonic RF phase shifter based on optical carrier and RF modulation sidebands amplitude and phase control," J. Lightw. Technol., vol. 30, no. 23, pp. 3672-3678, Dec. 2012.

[10] M. Pagani, D. Marpaung, and B. J. Eggleton, "Ultra-wideband microwave photonic phase shifter with configurable amplitude response," Opt. Lett., vol. 39 , no. 20, pp. 5854-5857, Oct. 2014.

[11] M. Burla et al., "On-chip programmable ultra-wideband microwave photonic phase shifter and true time delay unit," Opt. Lett., vol. 39, no. 21, pp. 6181-6184, Nov. 2014.

[12] J. Tang et al., "High-speed tunable broadband microwave photonics phase shifter based on an active microring resonator," in Proc. 25th Wireless Opt. Commun. Conf., Chengdu, China, May 2016, pp. 1-3.

[13] V. J. Urick et al., "Microwave phase shifting using coherent photonic integrated circuits," IEEE J. Sel. Top. Quantum Electron., vol. 22, no. 6, Nov./Dec. 2016, Art. no. 6100408.

[14] R. A. Soref and B. R. Bennett, "Electrooptical effects in silicon," IEEE J. Quantum Electron., vol. 23, no. 1, pp. 123-129, Jan. 1987.

[15] R. Bonjour et al., "Continuously tunable true-time delays with ultra-low settling time," Opt. Express, vol. 23, no. 5, pp. 6952-6964, Mar. 2015.

[16] Y. Liu et al., "Gigahertz optical tuning of an on-chip radio frequency photonic delay line," Optica, vol. 4, no. 4, pp. 418-423, Apr. 2017.

[17] "Europractice multi project wafer." [Online]. Available: http://www. europractice-ic.com/SiPhotonics_technology_imec_ISIPP25G.php

[18] K. Oda, N. Takato, H. Toba, and K. Nosu, "A wide-band guided-wave periodic multi/demultiplexer with a ring resonator for optical FDM transmission systems," J. Lightw. Technol., vol. 6, no. 6, pp. 1016-1023, Jun. 1988.

[19] K. Jinguji and M. Oguma, "Optical half-band filters," J. Lightw. Technol., vol. 18, no. 2, pp. 252-259, Feb. 2000.

[20] T. Kawanishi and M. Izutsu, "Linear single-sideband modulation for highSNR wavelength conversion," IEEE Photon. Technol. Lett., vol. 16, no. 6, pp. 1534-1536, Jun. 2004

[21] Y. Niu, Y. Li, D. Jin, L. Su, and A. V. Vasilakos, "A survey of millimeter wave communications (mmWave) for 5G: Opportunities and challenges," Wireless Netw., vol. 21, pp. 2657-2676, Apr. 2015.

[22] P. Dong et al., "GHz-bandwidth optical filters based on high-order silicon ring resonators," Opt. Express, vol. 18, no. 23, pp. 23784-23789, Nov. 2010.

[23] C. K. Madsen and J. H. Zhao, Optical Filter Design and Analysis. New York, NY, USA: Wiley, 1999.

[24] C. Porzi et al., "On the performance of advanced integrated microring filters for switching applications in next-generation elastic optical networks," Photon. Netw. Commun., vol. 31, no. 3, pp. 503-513, Jan. 2016.

[25] C. Porzi et al., "Integrated SOI high-order phase-shifted Bragg grating for microwave photonics signal processing," J. Lightw. Technol., vol. 35, no. 20, pp. 4479-4487, Oct. 2017.

[26] E. Timurdogan, C. V. Poulton, M. J. Byrd, and M. R. Watts, "Electric fieldinduced second-order nonlinear optical effects in silicon waveguides," Nature Photon., vol. 11, no. 3, pp. 200-208, Feb. 2017.

Authors' biographies not available at the time of publication. 